Argonne

ANL/SSS-18/3

\title{
A Model of Airborne Particle Removal by a Liquid Drop Spray
}

Strategic Security Sciences Division 


\begin{abstract}
About Argonne National Laboratory
Argonne is a U.S. Department of Energy laboratory managed by UChicago Argonne, LLC under contract DE-AC02-06CH11357. The Laboratory's main facility is outside Chicago, at 9700 South Cass Avenue, Argonne, Illinois 60439. For information about Argonne and its pioneering science and technology programs, see www.anl.gov.
\end{abstract}

\title{
DOCUMENT AVAILABILITY
}

Online Access: U.S. Department of Energy (DOE) reports produced after 1991 and a growing number of pre-1991 documents are available free via DOE's SciTech Connect (http://www.osti.gov/scitech/).

Reports not in digital format may be purchased by the public from the National Technical Information Service (NTIS):

U.S. Department of Commerce

National Technical Information Service

5301 Shawnee Road

Alexandria, VA 22312

www.ntis.gov

Phone: (800) 553-NTIS (6847) or (703) 605-6000

Fax: (703) 605-6900

Email: orders@ntis.gov

Reports not in digital format are available to DOE and DOE contractors from:

U.S. Department of Energy

Office of Scientific and Technical Information

P.O. Box 62

Oak Ridge, TN 37831-0062

\section{Disclaimer}

This report was prepared as an account of work sponsored by an agency of the United States Government. Neither the United States Government nor any agency thereof, nor UChicago Argonne, LLC, nor any of their employees or officers, makes any warranty, express or implied, or assumes any legal liability or responsibility for the accuracy, completeness, or usefulness of any information, apparatus, product, or process disclosed, or represents that its use would not infringe privately owned rights. Reference herein to any specific commercial product, process, or service by trade name, trademark, manufacturer, or otherwise, does not necessarily constitute or imply its endorsement, recommendation, or favoring by the United States Government or any agency thereof. The views and opinions of document authors expressed herein do not necessarily state or reflect those of the United States Government or any agency thereof, Argonne National Laboratory, or UChicago Argonne, LLC. 


\section{A Model of Airborne Particle Removal by a Liquid Drop Spray}

by

James C. Liljegren

Strategic Security Sciences Division, Argonne National Laboratory

August 2018 
This page intentionally left blank. 


\section{Contents}

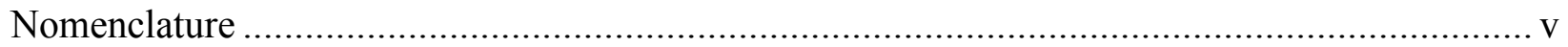

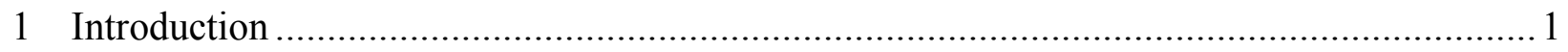

2 Particle Removal by a Liquid Drop Spray ........................................................ 3

3 Single-Drop Collection Efficiency ........................................................................ 5

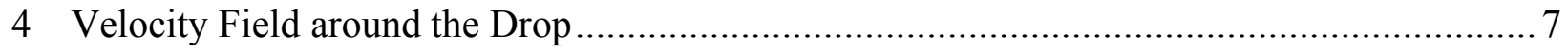

4.1 Formulation of the Problem............................................................................ 7

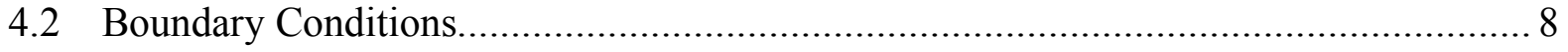

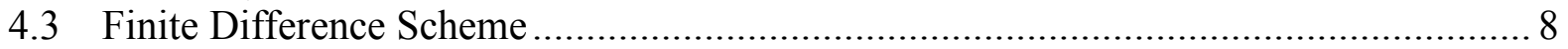

4.4 Solution of the Finite Difference Equations .................................................... 10

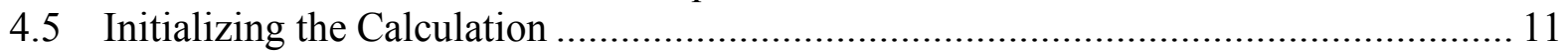

4.6 Verification and Validation of the Stream Function Calculations ........................... 12

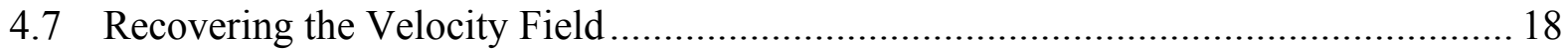

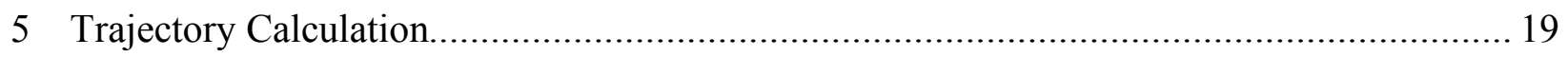

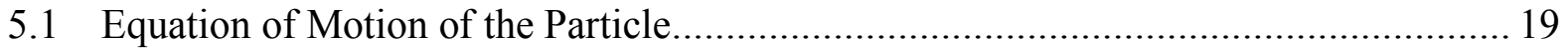

5.2 Electrostatic Force on the Particle ..................................................................... 20

5.3 Phoretic Forces on the Particle .......................................................................... 21

5.4 Finding the Fluid Velocity at the Particle Position ........................................... 22

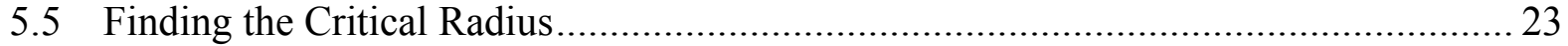

5.6 Results of the Trajectory Calculations .......................................................... 23

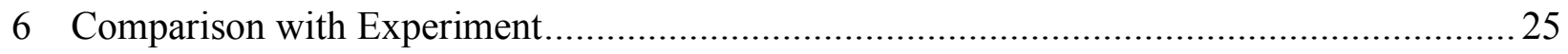

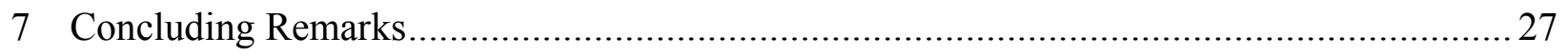

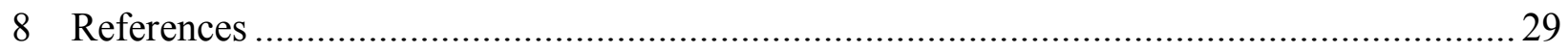




\section{Figures}

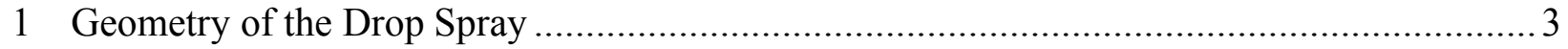

2 Definition of Critical Radius and Collision Efficiency........................................................ 5

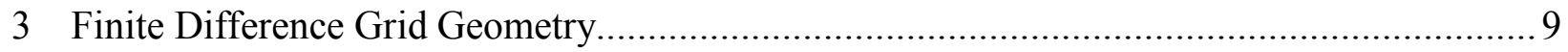

4 Calculated Surface Pressure Distributions around a Falling Drop ....................................... 15

5 Calculated Surface Vorticity Distributions around a Falling Drop ….................................. 15

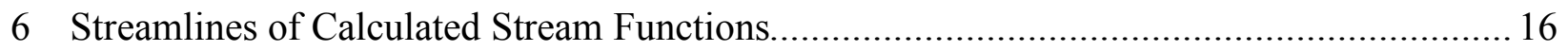

7 Drag Coefficients from the Present Work Compared with a Fit to Experimental Data

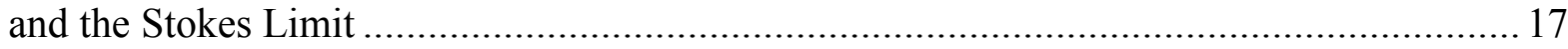

8 Trajectories and Collision Efficiencies for a 42- $\mu \mathrm{m}$-Diameter Drop and 2- $\mu \mathrm{m}$-Diameter Particle for Several Combinations of Electrostatic Charge..................................................2 24

\section{Tables}

1 Values of the Coefficients for Large Viscosity Ratio ......................................................... 12

2 Parameters and Derived Results of the Stream Function Calculations.................................. 13

3 Calculated Filter Efficiencies for Comparison with a Knockdown Spray Experiment..........25 


\section{Nomenclature}

$A_{d} \quad$ cross-sectional area swept by a falling drop

$A_{t} \quad$ cross-sectional area of a subway tunnel or drop spray

$A_{1}, A_{2}, A_{3}, A_{4}, B_{1}, B_{2}, B_{3}, B_{4}$ coefficients in the trial stream function of Hamielec et al. (1963)

$C_{d} \quad$ drop mass concentration

$C_{D} \quad$ drag coefficient (total)

$C_{D P} \quad$ drag coefficient due to pressure (form drag)

$C_{D F} \quad$ drag coefficient due to shear (friction)

$C_{D}^{a}$

drag coefficient for $N_{R e} \ll 1$ from the analytical expression for a bounded flow

$C_{\mathrm{sc}} \quad$ Cunningham slip correction factor

$d_{d} \quad$ drop diameter

$\bar{d} \quad$ mean spacing between drops

$E \quad$ collection efficiency (collision efficiency)

$E_{f} \quad$ filter efficiency

$F_{E} \quad$ electrostatic force

$F_{j} \quad$ electrostatic force coefficients

$F \quad$ vorticity function, $=\zeta / r \sin \theta$

$G \quad$ vorticity function, $=\zeta r \sin \theta$

$g \quad$ gravitational acceleration

net gravitational acceleration accounting for buoyancy of air

$H$

height of the drop spray

K

Stokes number $=\left(r_{p} / r_{d}\right)^{2} \rho_{p} \mathrm{~N}_{\mathrm{Re}} \mathrm{C}_{\mathrm{sc}} /(9 \rho)$

$L$

length (depth) of drop spray

$M_{p} \quad$ mass of particles

$m_{d}$

mass of a single drop

$N$

number concentration

$N_{0}$

initial number concentration

$N_{d}$

drop number concentration

$N_{p}$

particle number concentration

$N_{r}$

number of radial grid points

$N_{\theta}$

number of polar grid points

$N_{R e}$

Reynolds number $=\rho U_{\infty} d_{d} / \mu$

$\mathrm{N}_{\mathrm{Fr}}$

Froude number $=U_{\infty}^{2} /\left(g^{*} r_{d}\right)$

$p$

dynamic pressure (dimensionless)

$p_{0}$

stagnation pressure (dimensionless)

$p_{\infty}^{\prime}$

free stream static pressure (dimensional)

$Q_{d}$

charge on a drop

$Q_{p}$

charge on a particle

$q_{d}$

charge density on a drop

$q_{p}$

charge density on a particle

$\hat{\boldsymbol{r}} \quad$ unit vector directed from the center of the drop to the center of the particle 
$r$

$r_{c}$

$r_{d}$

$r_{p}$

$r_{\infty}$

$t$

U

$U$

$U_{\infty}$

V

V

$V_{\infty}$

$\mathcal{V}$

$\mathcal{V}_{d}$

$\bar{v}$

$v_{f}$

$\mathcal{V}_{S}$

$\dot{v}_{\text {air }}$

$\dot{\mathcal{V}}_{\text {liq }}$

$X$

$y$

$x_{p}, y_{p}$

$x_{p 0}, y_{p 0}$

$Z$

$\widehat{\mathbf{z}}$

$\Delta \theta$

$\Delta z$

$\delta \theta$

$\delta z$

$\epsilon$

$\lambda$

$\rho$

$\rho_{d}$

$\rho_{p}$

$\mu$

$\theta$

$\theta_{p}, z_{p}$

$\psi$

$\zeta$

$\omega_{\psi}$

$\omega_{\mathrm{G}}$ radial dimension

critical radius

drop radius

particle radius

outer boundary radius

contact time

fluid velocity vector

fluid flow speed

terminal fall speed of a drop

particle velocity vector

particle speed

terminal fall speed of a particle

volume

volume of a single drop

mean volume occupied by a single drop

filtered volume

volume of the spray

volume flow rate of air

volume flow rate of liquid

rectilinear dimension parallel to free stream flow

rectilinear dimension perpendicular to free stream flow

particle location in rectilinear coordinates

initial particle location

transformed radial dimension

unit vector in the direction of gravity

polar grid spacing

transformed radial grid spacing

polar interpolation distance

transformed radial interpolation distance

dielectric constant of air

particle removal rate

density of air

density of a drop

density of a particle

viscosity of air

polar dimension

particle location in transformed spherical polar coordinates

stream function

vorticity

relaxation factor for stream function calculation

relaxation factor for $G$ function calculation 


\section{Introduction}

The removal of airborne particles by liquid drops is an important atmospheric and industrial process. In the atmospheric science literature, it is variously referred to as "precipitation scavenging," "wet deposition," or "rainout" and has been extensively studied for its role in the formation of clouds and the removal of atmospheric pollutants (Pruppacher et al. 1983; Slinn 1984; Pruppacher and Klett 2010). Engineered systems of water sprays, or "knockdown sprays," have been developed for controlling airborne dust, particularly in mining applications to mitigate potential health impacts of respirable particles as well as the potential for dust explosions (Cheng 1973; Schowengerdt and Brown 1976; Prostanski 2013).

Recently, it has been shown that knockdown sprays of electrically charged liquid drops can be effective in neutralizing and removing chemical and biological agents released in a confined space (Betty et al. 2010; Tucker et al. 2016), and that they may be suitable for mitigating the spread of chemical and biological agents released in subway systems. The performance of knockdown sprays depends on several parameters, including the sizes of the drops and particles, static electric charges on the drops and particles, drop concentrations, and the geometry of the spray. The model described herein was developed to estimate the potential performance of a knockdown spray in terms of these parameters.

The geometry of the drop spray is presented first, followed by a description of the modeling approach in which the spray is treated as a filter. The filter efficiency is derived from the particle removal rate, which requires that the single drop collection efficiency be determined. The calculation of the single drop collection efficiency comprises two main tasks, which are presented in succession: calculating the flow field around a falling drop, which requires the solution of the Navier-Stokes equations, and then calculating the trajectory of a particle in that flow field subject to inertial, gravitational, and electrostatic forces. The model development is then followed by a comparison with the results of a laboratory investigation of an electrically charged drop spray. Despite uncertainties in some of the test parameters, the comparison suggests that the drop spray model may be able to accurately estimate the performance of a charged knockdown spray, and that the model may be useful in refining the design of such a spray for mitigation of a biological agent release in an indoor environment. 
This page intentionally left blank. 


\section{Particle Removal by a Liquid Drop Spray}

The geometry of the drop spray considered here is shown in Fig. 1. The airflow in a subway tunnel with speed $U$ moves a volume of air $\mathcal{V}_{f}$ having a particle mass concentration $C_{p}$ through the drop spray, which behaves as a filter with efficiency $E_{f}$ for particles of a given size.

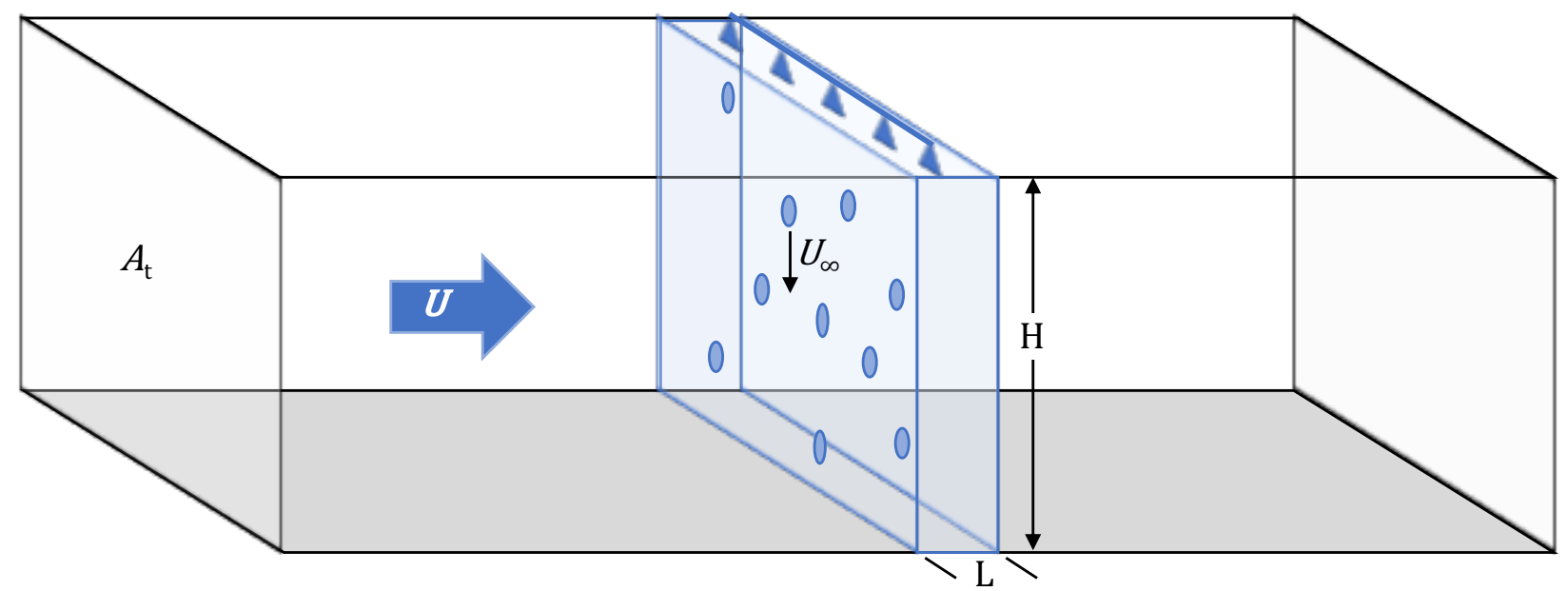

Figure 1 Geometry of the Drop Spray

The mass of particles (of a given size) removed is then

$$
M_{p}=C_{p} \mathcal{V}_{f} E_{f}
$$

The filtered volume is determined from the cross-sectional area of the tunnel (or spray) $A_{t}$ and the flow speed through that cross-section during the model time step: $\mathcal{V}_{f}=A_{t} U \Delta t$. For the case of no flow $(U=0), \mathcal{V}_{f}=A_{t} L$, where $L$ is the depth of the drop spray. In the latter case, the same volume would be repeatedly filtered and the particle mass concentration would rapidly decrease to zero.

The filter efficiency is found by integrating the rate of change of the particle number concentration $d N_{p} / d t=-\lambda N_{p}$ over the contact time $t$

$$
\log \frac{N}{N_{0}}=\int_{0}^{t}-\lambda d t^{\prime} .
$$

Then

$$
E_{f}=1-N / N_{0}=1-e^{-\lambda t}
$$


where $\lambda=E N_{d} A_{d} U_{\infty}$ is the particle removal rate; $N_{d}$ is the drop number concentration; $A_{d}=$ $\pi\left(r_{d}+r_{p}\right)^{2}$ is the cross-sectional area swept by the falling drop having the radius $r_{d}$ with the particle radius $r_{p} ; U_{\infty}$ is the terminal settling speed of the drop; and $E$ is the single-drop collection efficiency. The contact time is the time the drops are airborne, i.e., the time it takes the drops to fall the height of the spray $H$ at their terminal settling speed: $t=H / U_{\infty}$. Combining the expressions for the removal rate and contact time, the filter efficiency becomes

$$
E_{f}=1-e^{-E N_{d} A_{d} H}
$$

This may also be expressed in terms of the volumetric flow rate of the drop liquid $\dot{\mathcal{V}}_{\text {liq }}$ by recognizing that

$$
N_{d}=\frac{\dot{\mathcal{V}}_{\text {liq }}}{\mathcal{V}_{d}\left(\mathcal{V}_{s} / t\right)}=\frac{\dot{\mathcal{V}}_{\text {liq }}}{\mathcal{V}_{d} \dot{\mathcal{V}}_{\text {air }}}
$$

where $\mathcal{V}_{d}=\frac{4}{3} \pi r_{d}^{3}$ is the volume of a single drop, $\mathcal{V}_{s}=A_{t} L$ is the volume of air occupied by the spray, and $\dot{\mathcal{V}}_{\text {air }}=\left(\mathcal{V}_{s} / t\right)=A_{t} L U_{\infty} / H$ is the rate at which that volume is filled by the drops as they fall. Because the particles are typically much smaller than the drops, the simplification $A_{d}=$ $\pi r_{d}^{2}$ may be combined with this expression for $N_{d}$ to express the filter efficiency as

$$
E_{f}=1-e^{-3 E H \dot{v}_{l i q} / 4 r_{d} \dot{v}_{a i r}}
$$

which appears in the applied drop spray literature (Cheng 1973 and references therein). 


\section{Single-Drop Collection Efficiency}

As illustrated in Fig. 2, the single-drop collision efficiency is

$$
E=\frac{\pi r_{c}^{2}}{\pi\left(r_{d}+r_{p}\right)^{2}}
$$

where $r_{c}$ is the critical radius: the maximum offset from the centerline of the drop that a particle trajectory could start from and still collide with the drop. A particle collides with the drop when the separation between their centers is equal to the sum of their radii: $r_{d}+r_{p}$. Particles that approach the drop from within the critical radius will collide with the drop; particles that approach from outside the critical radius will miss the drop. It is typically assumed (e.g., Beard and Grover 1974) that particles that collide with the drop will adhere to it so that the collection efficiency is usually taken to be equal to the collision efficiency.

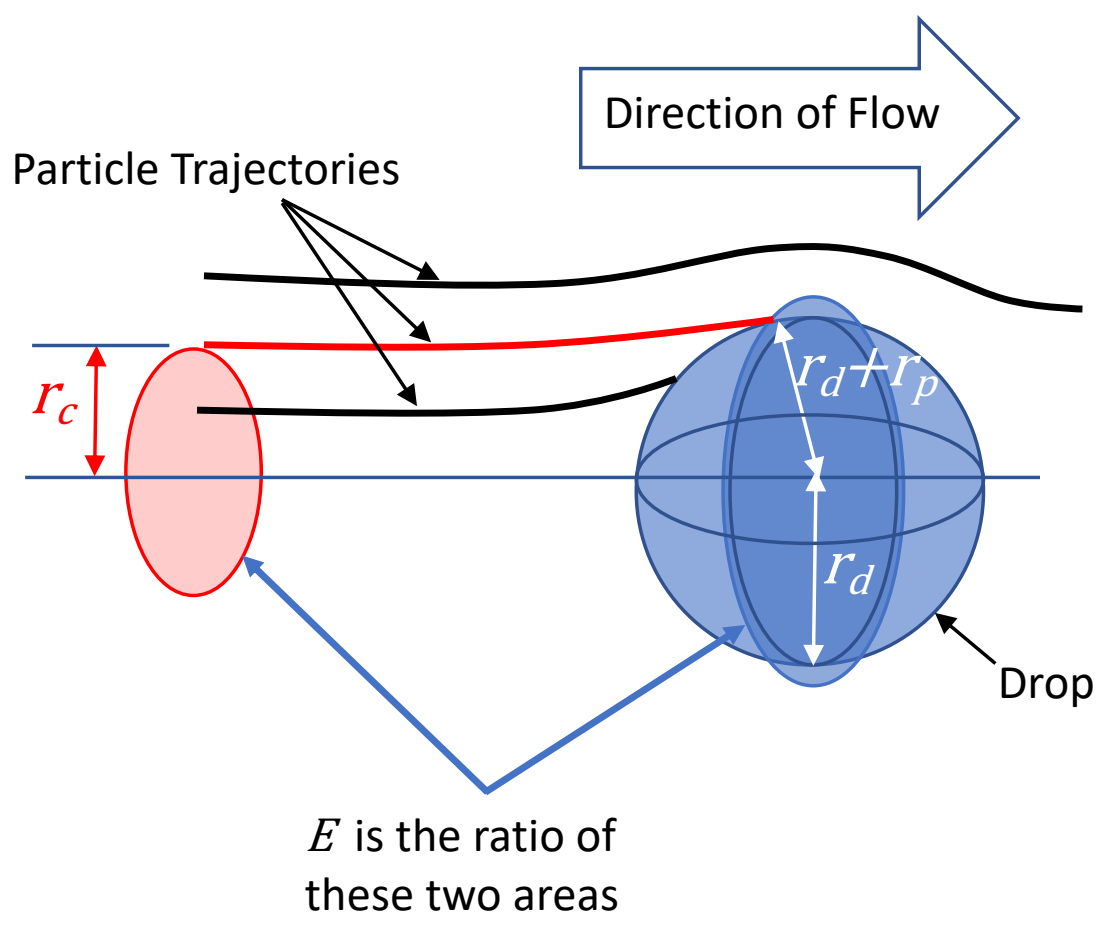

Figure 2 Definition of Critical Radius and Collision Efficiency 
This page intentionally left blank. 


\section{Velocity Field around the Drop}

\subsection{Formulation of the Problem}

To calculate the trajectory of the particle and thereby determine the critical radius, the velocity field around the drop falling at terminal velocity must first be calculated. For values of the drop Reynolds number $N_{R e} \ll 1$, where $N_{R e}=\rho U_{\infty} d_{d} / \mu$, and $\rho$ and $\mu$ are the density and viscosity of air, respectively, the inertia terms of the Navier-Stokes equation may be neglected and an analytical solution for the flow around a sphere is possible ("creeping flow" or "Stokes flow"). Unless the drops are less than about $20 \mu \mathrm{m}$ in diameter, this solution is invalid.

Jenson (1959) solved the Navier-Stokes equation for viscous, incompressible flow around a sphere for $N_{R e} \leq 40$ using a finite difference method on a mechanical desktop calculator. Hamielec et al. (1967) extended Jenson's work to $N_{R e}=100$ using a digital computer, and LeClaire et al. (1970) further extended the solutions to $N_{R e}=400$; their approach has been followed here.

Begin with the Navier-Stokes equation in terms of the stream function ${ }^{1} \psi$ in spherical polar coordinates $(r, \theta)$

$$
\frac{N_{R e}}{2}\left[\frac{\partial \psi}{\partial r} \cdot \frac{\partial}{\partial \theta}\left(\frac{E^{2} \psi}{r^{2} \sin ^{2} \theta}\right)-\frac{\partial \psi}{\partial \theta} \cdot \frac{\partial}{\partial r}\left(\frac{E^{2} \psi}{r^{2} \sin ^{2} \theta}\right)\right] \sin \theta=E^{4} \psi,
$$

where the operator $E^{2}$ is

$$
E^{2}=\frac{\partial^{2}}{\partial r^{2}}+\frac{\sin \theta}{r^{2}} \frac{\partial}{\partial \theta}\left(\frac{1}{\sin \theta} \frac{\partial}{\partial \theta}\right)
$$

Split the Navier-Stokes equation into two simultaneous second-order equations by introducing the vorticity $\zeta$

$$
\begin{gathered}
E^{2} \Psi=\zeta \mathrm{r} \sin \theta \\
\frac{N_{R e}}{2}\left[\frac{\partial \psi}{\partial r} \cdot \frac{\partial}{\partial \theta}\left(\frac{\zeta}{\mathrm{r} \sin \theta}\right)-\frac{\partial \psi}{\partial \theta} \cdot \frac{\partial}{\partial r}\left(\frac{\zeta}{\mathrm{r} \sin \theta}\right)\right] \sin \theta=E^{2}(\zeta \mathrm{r} \sin \theta)
\end{gathered}
$$

The velocity components are recovered by differentiating the stream function:

1 The stream function is convenient because it satisfies the continuity equation identically, thereby reducing the number of equations to be solved. Physically, there is no mass flow across lines of constant $\psi$ ("streamlines"); the difference between the values of $\psi$ for two streamlines is equal to the mass flow between them (White 1974). 


$$
U_{\theta}=\frac{1}{\mathrm{r} \sin \theta} \frac{\partial \psi}{\partial r} ; U_{r}=\frac{-1}{r^{2} \sin \theta} \frac{\partial \psi}{\partial \theta}
$$

All quantities have been non-dimensionalized using the terminal fall speed and radius of the drop:

$$
r=\frac{r^{\prime}}{r_{d}} ; \psi=\frac{\psi^{\prime}}{U_{\infty} r_{d}^{2}} ; \zeta=\frac{\zeta^{\prime} r_{d}}{U_{\infty}} ; U_{r}=\frac{U_{r}^{\prime}}{U_{\infty}} ; U_{\theta}=\frac{U_{\theta}^{\prime}}{U_{\infty}} ;
$$

where the dimensional quantities are indicated by a prime (').

\subsection{Boundary Conditions}

For non-evaporating drops (no radial mass efflux from the drop), the boundary conditions required to solve the above equations are as follows.

Along the axis of symmetry $\left(\theta=0^{\circ}\right.$ and $\left.\theta=180^{\circ}\right)$

$$
\psi=0, \zeta=0 .
$$

On the surface of the sphere $(r=1)$

$$
\psi=0, \quad \zeta=\frac{E^{2} \Psi}{\sin \theta} .
$$

The vorticity at the surface of the sphere must be computed by recognizing that the tangential velocity at the sphere surface is zero (i.e., the no-slip condition), $\partial \psi / \partial r=0$.

Far from the sphere $\left(r=r_{\infty}\right)$ the flow is parallel and irrotational

$$
\psi=\frac{1}{2} r_{\infty}^{2} \sin ^{2} \theta, \zeta=0 .
$$

\subsection{Finite Difference Scheme}

Jenson (1959) derived the finite difference scheme for the stream function and vorticity at the interior grid points by a Taylor-series expansion accurate to second order. He also carried out experiments with fourth-order expansions to verify the sufficiency of the second-order scheme for $N_{R e}=5$. Recognizing that the stream function and vorticity variations are largest near the sphere and smallest far from it, Jenson implemented a coordinate transformation $\left(r=e^{z}\right)$, using 
constant intervals $\Delta z$ and $\Delta \theta$ to provide an exponential grid spacing in the radial direction. The grid design is depicted in Fig. 3.

The transformed equations for vorticity and stream function then become

$$
e^{2 z} E^{2} \psi-\zeta e^{3 z} \sin \theta=0
$$

and

$$
\frac{N_{R e}}{2}\left[\frac{\partial \psi}{\partial z} \cdot \frac{\partial F}{\partial \theta}-\frac{\partial \psi}{\partial \theta} \cdot \frac{\partial F}{\partial z}\right] e^{z} \sin \theta-e^{2 z} E^{2} \mathrm{G}=0
$$

where

$$
e^{2 z} E^{2}=\frac{\partial^{2}}{\partial z^{2}}-\frac{\partial}{\partial z}+\sin \theta \frac{\partial}{\partial \theta}\left(\frac{1}{\sin \theta} \frac{\partial}{\partial \theta}\right)
$$

and

$$
F=\frac{\zeta}{e^{z} \sin \theta} ; G=\zeta e^{z} \sin \theta
$$

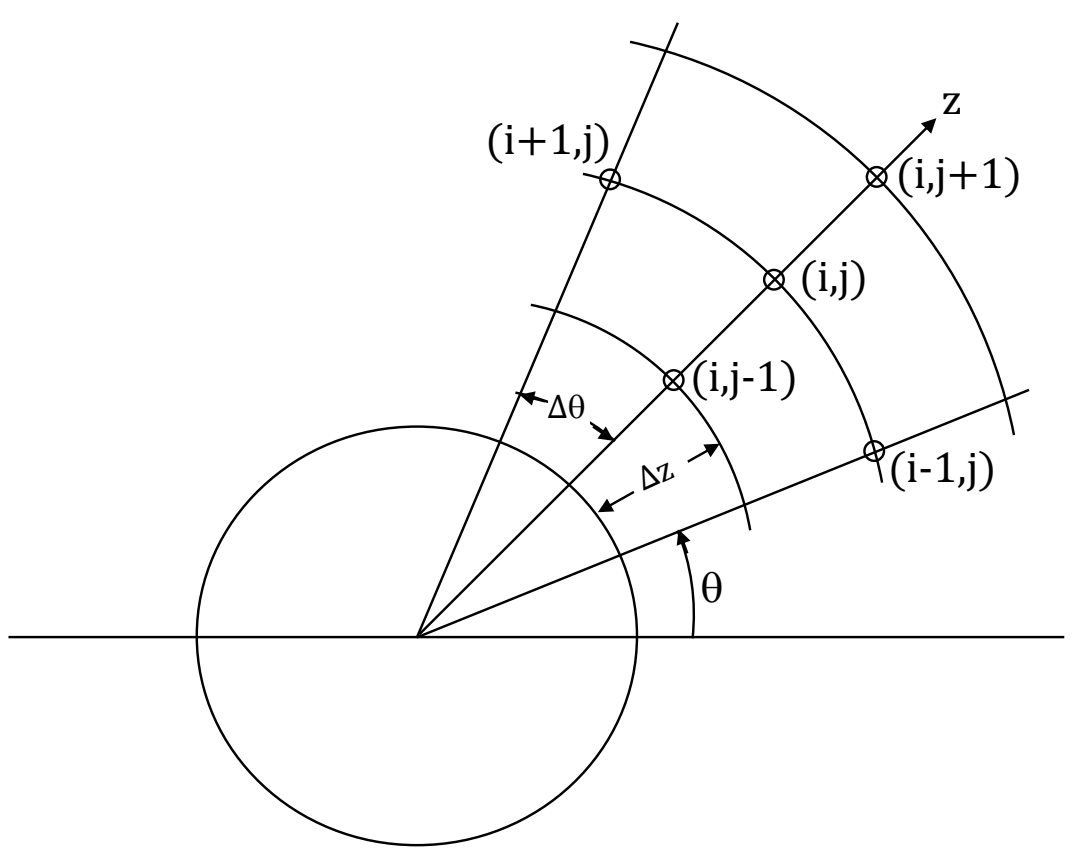

Figure 3 Finite Difference Grid Geometry 
In finite difference form, these become

$$
\begin{aligned}
& \left(\frac{2}{\Delta z^{2}}+\frac{2}{\Delta \theta^{2}}\right) \Psi_{i, j}=\left(\frac{2-\Delta z}{2 \Delta z^{2}}\right) \psi_{i, j+1}+\left(\frac{2+\Delta z}{2 \Delta z^{2}}\right) \psi_{i, j-1}+\left(\frac{2-\Delta \theta \cot \theta_{i}}{2 \Delta \theta^{2}}\right) \psi_{i+1, j}+\left(\frac{2+\Delta \theta \cot \theta_{i}}{2 \Delta \theta^{2}}\right) \psi_{i-1, j}- \\
& G_{i, j} e^{2 z_{j}}
\end{aligned}
$$

and

$$
\begin{aligned}
& \left(\frac{2}{\Delta z^{2}}+\frac{2}{\Delta \theta^{2}}\right) \mathrm{G}_{i, j}=\left(\frac{2-\Delta z}{2 \Delta z^{2}}\right) \mathrm{G}_{i, j+1}+\left(\frac{2+\Delta z}{2 \Delta z^{2}}\right) \mathrm{G}_{i, j-1}+\left(\frac{2-\Delta \theta \cot \theta_{i}}{2 \Delta \theta^{2}}\right) \mathrm{G}_{i+1, j}+\left(\frac{2+\Delta \theta \cot \theta_{i}}{2 \Delta \theta^{2}}\right) \mathrm{G}_{i-1, j}- \\
& \frac{N_{R e}}{4} e^{z_{j}} \sin \theta_{i}\left[\left(\frac{\Psi_{i, j+1}-\Psi_{i, j-1}}{2 \Delta z}\right)\left(\frac{\mathrm{F}_{i+1, j}-\mathrm{F}_{i-1, j}}{\Delta \theta}\right)-\left(\frac{\Psi_{i+1, j}-\Psi_{i-1, j}}{2 \Delta \theta}\right)\left(\frac{\mathrm{F}_{i, j+1}-\mathrm{F}_{i, j-1}}{\Delta z}\right)\right] .
\end{aligned}
$$

The boundary condition on the vorticity at the surface of the sphere yields

$$
G_{i, 1}=\frac{-7 \psi_{i, 1}+8 \psi_{i, 2}-\psi_{i, 3}}{2 \Delta z^{2}} ; F_{i, 1}=\frac{G_{i, 1}}{\sin ^{2} \theta_{i}}
$$

\subsection{Solution of the Finite Difference Equations}

Through an analysis of residuals, Jenson (1959) developed a criterion for the radial grid spacing: $\Delta z<4 / N_{R e}$ and used values that were half of this limit (0.4-0.05); because of his limited computational resources, he used an angular grid spacing of $12^{\circ}$. Hamielec et al. (1967) and LeClaire et al. (1970) used $\Delta z=0.05$ or 0.025 , and $\Delta \theta=3^{\circ}$. For the present work, $\Delta \mathrm{z}=0.025$ and $\Delta \theta=2^{\circ}$ primarily to provide a more dense grid of velocity components for subsequent interpolation during particle trajectory calculations. Higher resolutions can be used, if necessary.

The results are sensitive to the position of the outer boundary $\left(r=r_{\infty}\right)$. Jenson (1959) used the $r_{\infty}=6$ due to computational limitations, which resulted in larger drag coefficients than values experimentally obtained, particularly at small Reynolds numbers (White 1974, p. 227). Hamielec et al. (1967) obtained agreement between calculated and experimental drag coefficients within $8 \%$ using $r_{\infty}$ ranging from 19 at $N_{R e}=0.1$ to 7 at $N_{R e}=100$. LeClaire et al. (1970) experimented with a wide range of values of $r_{\infty}$. The values of $r_{\infty}$ used in the present work are listed in Table 2 and discussed along with the results below.

With the grid spacing and position of the outer boundary in hand, the finite difference calculations begin with the first interior row of the grid closest to the sphere, starting near the forward stagnation point and proceeding toward the rear stagnation point, followed by subsequent rows working toward the outer boundary. In this way, new information is propagated around the sphere and radially outward as would be the case for an actual falling drop (Anderson et al. 1984, pp. 130-136; White 1974, p. 221). The solution proceeds iteratively using a relaxation approach: 


$$
\begin{gathered}
\Psi_{i, j}^{n+1}=\Psi_{i, j}^{n}+\omega_{\psi}\left(\Psi_{i, j}^{*}-\Psi_{i, j}^{n}\right) ; \\
G_{i, j}^{n+1}=G_{i, j}^{n}+\omega_{G}\left(G_{i, j}^{*}-G_{i, j}^{n}\right),
\end{gathered}
$$

where the superscript indicates the iteration, the asterisk indicates the values calculated from the finite difference equations using values from the $n^{\text {th }}$ iteration, and $\omega$ is a relaxation factor less than 1. Based on numerical experiments carried out by LeClaire et al. (1970), a value of $\omega_{\psi}=1.0$ and $\omega_{\mathrm{G}}=0.1$ for $N_{R e} \leq 200$ was used in the present work. Iteration continues until $\psi^{n+1}-\psi^{n}<$ $10^{-5}$ and $\mathrm{G}^{n+1}-\mathrm{G}^{n}<10^{-5}$ at all grid points.

\subsection{Initializing the Calculation}

Initial values of $\psi, F$, and $G$ were determined from the approximate stream functions derived by Hamielec et al. (1963) by applying the Galerkin method to find the coefficients of a trial stream function:

$$
\begin{gathered}
\Psi=(A+B \cos \theta) \sin ^{2} \theta \\
A=\left(\frac{r^{2}}{2}+\frac{A_{1}}{r}+\frac{A_{2}}{r^{2}}+\frac{A_{3}}{r^{3}}+\frac{A_{4}}{r^{4}}\right) ; B=\left(\frac{B_{1}}{r}+\frac{B_{2}}{r^{2}}+\frac{B_{3}}{r^{3}}+\frac{B_{4}}{r^{4}}\right) .
\end{gathered}
$$

For a liquid drop falling in air, such that the ratio of the viscosity of the liquid to that of air is large, the coefficients are

$$
\begin{aligned}
A_{2}= & (-120 / 29)+(-75 / 29) A_{1} ; \\
A_{3}= & (153 / 29)+(63 / 29) A_{1} ; \\
A_{4}= & (-47.5 / 29)+(-17 / 29) A_{1} ; \\
& B_{2}=(-69 / 27) B_{1} ; \\
& B_{3}=(57 / 27) B_{1} ; \\
& B_{4}=(-15 / 27) B_{1} .
\end{aligned}
$$

$A_{1}$ and $B_{1}$ are listed in Table 1. 
Table 1 Values of the Coefficients for Large Viscosity Ratio

\begin{tabular}{crr}
\hline$N_{R e}$ & $A_{1}$ & \multicolumn{1}{l}{$B_{1}$} \\
\hline$<<1$ & -4.5000 & 0 \\
10 & -4.4785 & -0.9208 \\
20 & -4.4145 & -1.8494 \\
30 & -4.3086 & -2.7935 \\
40 & -4.1621 & -3.7603 \\
50 & -3.9771 & -4.7560 \\
60 & -3.7564 & -5.7851 \\
70 & -3.5038 & -6.8502 \\
80 & -3.2242 & -7.9504 \\
90 & -2.9235 & -9.0818 \\
100 & -2.6084 & -10.2364 \\
200 & 0.1829 & -20.6800 \\
300 & 1.6282 & -26.8219 \\
400 & 2.3846 & -30.3244 \\
500 & 2.8384 & -32.5324 \\
\hline
\end{tabular}

Then

$$
\begin{aligned}
G & =E^{2} \Psi=\frac{\partial^{2} \Psi}{\partial r^{2}}+\frac{\sin \theta}{r^{2}} \frac{\partial}{\partial \theta}\left(\frac{1}{\sin \theta} \frac{\partial \psi}{\partial \theta}\right) \\
& =\left[\frac{\partial^{2} \mathrm{~A}}{\partial r^{2}}-\frac{2 A}{r^{2}}+\left(\frac{\partial^{2} \mathrm{~B}}{\partial r^{2}}-\frac{6 B}{r^{2}}\right) \cos \theta\right] \sin ^{2} \theta ; \\
& =\left[\left(\frac{4 A_{2}}{r^{4}}+\frac{10 A_{3}}{r^{5}}+\frac{18 A_{4}}{r^{6}}\right)+\left(\frac{-4 B_{1}}{r^{3}}+\frac{6 B_{3}}{r^{5}}+\frac{14 B_{4}}{r^{6}}\right) \cos \theta\right] \sin ^{2} \theta ;
\end{aligned}
$$

and

$$
F=\frac{G}{r^{2} \sin ^{2} \theta}=\frac{1}{r^{2}}\left[\left(\frac{4 A_{2}}{r^{4}}+\frac{10 A_{3}}{r^{5}}+\frac{18 A_{4}}{r^{6}}\right)+\left(\frac{-4 B_{1}}{r^{3}}+\frac{6 B_{3}}{r^{5}}+\frac{14 B_{4}}{r^{6}}\right) \cos \theta\right] .
$$

\subsection{Verification and Validation of the Stream Function Calculations}

Parameters used to calculate the stream functions for several values of the Reynolds number along with drag coefficients and pressures at the forward and rearward stagnation points derived from the results are presented in Table 2. The results compare well with those in a similar table presented by LeClaire et al. (1970). 
Table 2 Parameters and Derived Results of the Stream Function Calculations

\begin{tabular}{|c|c|c|c|c|c|c|c|c|c|c|c|}
\hline$N_{R e}$ & $\Delta z$ & $\Delta \theta\left(^{\circ}\right)$ & $r_{\infty}$ & $\omega_{\psi}$ & $\omega_{\mathrm{G}}$ & $C_{D P}$ & $C_{D F}$ & $C_{D}$ & $C_{D}^{a}$ & $P_{o}$ & $P_{\pi}$ \\
\hline 0.01 & 0.025 & 2 & 1808 & 1 & 1 & 801.807 & 1606.592 & 2408.399 & 2402.391 & 594.953 & -607.842 \\
\hline 0.01 & 0.025 & 2 & 90 & 1 & 1 & 815.586 & 1634.502 & 2450.088 & 2448.967 & 605.082 & -618.386 \\
\hline 0.01 & 0.025 & 2 & 90 & 1 & 0.1 & 803.286 & 1630.935 & 2434.221 & 2448.967 & 609.324 & -595.688 \\
\hline 0.1 & 0.025 & 2 & 992 & 1 & 0.1 & 79.927 & 162.854 & 242.781 & 240.436 & 61.873 & -58.026 \\
\hline 0.1 & 0.025 & 2 & 365 & 1 & 0.1 & 79.932 & 162.826 & 242.757 & 241.189 & 61.857 & -58.050 \\
\hline 0.1 & 0.025 & 2 & 90 & 1 & 1 & 82.022 & 164.369 & 246.390 & 244.897 & 61.760 & -61.282 \\
\hline 0.1 & 0.025 & 2 & 90 & 1 & 0.1 & 80.745 & 163.774 & 244.518 & 244.897 & 62.087 & -59.039 \\
\hline 0.1 & 0.025 & 2 & 20 & 1 & 0.1 & 87.139 & 175.122 & 262.26 & 263.589 & 65.545 & -65.174 \\
\hline 1 & 0.025 & 2 & 992 & 1 & 0.1 & 9.164 & 18.185 & 27.349 & 24.044 & 7.655 & -6.105 \\
\hline 1 & 0.025 & 2 & 90 & 1 & 0.1 & 9.165 & 18.188 & 27.353 & 24.490 & 7.656 & -6.106 \\
\hline 1 & 0.025 & 2 & 20 & 1 & 0.1 & 9.367 & 18.607 & 27.974 & 26.359 & 7.783 & -6.281 \\
\hline 5 & 0.025 & 2 & 90 & 1 & 0.1 & 2.448 & 4.704 & 7.152 & $\_^{\text {(a) }}$ & 2.580 & -1.181 \\
\hline 5 & 0.025 & 2 & 20 & 1 & 0.1 & 2.476 & 4.740 & 7.215 & - & 2.588 & -1.215 \\
\hline 10 & 0.025 & 2 & 20 & 1 & 0.1 & 1.537 & 2.803 & 4.34 & - & 1.862 & -0.606 \\
\hline 20 & 0.025 & 2 & 20 & 1 & 0.1 & 1.019 & 1.711 & 2.73 & - & 1.466 & -0.317 \\
\hline 30 & 0.025 & 2 & 20 & 1 & 0.1 & 0.827 & 1.296 & 2.123 & - & 1.325 & -0.233 \\
\hline 40 & 0.025 & 2 & 20 & 1 & 0.1 & 0.724 & 1.068 & 1.792 & - & 1.251 & -0.196 \\
\hline 50 & 0.025 & 2 & 20 & 1 & 0.1 & 0.656 & 0.921 & 1.577 & - & 1.206 & -0.176 \\
\hline 100 & 0.025 & 2 & 20 & 1 & 0.1 & 0.499 & 0.583 & 1.082 & - & 1.112 & -0.142 \\
\hline 100 & 0.025 & 2 & 12 & 1 & 0.1 & 0.503 & 0.584 & 1.086 & - & 1.112 & -0.142 \\
\hline 200 & 0.025 & 2 & 12 & 1 & 0.1 & 0.397 & 0.368 & 0.765 & - & 1.065 & -0.113 \\
\hline 300 & 0.025 & 2 & 12 & 1 & 0.05 & 0.347 & 0.279 & 0.627 & - & 1.048 & -0.086 \\
\hline 400 & 0.025 & 2 & 12 & 1 & 0.025 & 0.317 & 0.229 & 0.546 & - & 1.040 & -0.069 \\
\hline
\end{tabular}

(a) The analytical expression for $C_{D}$ in a bounded flow is valid only for small Reynolds numbers.

The drag coefficient is the drag force $F$ on the drop exerted by the fluid arising from both pressure (form drag) and shear stress (friction) normalized by the kinetic head (i.e., the dynamic pressure far from the drop) and cross-sectional area (White 1974, p. 206):

$$
C_{D}=\frac{F}{\left(\frac{1}{2} \rho U^{2}\right) \pi r_{d}^{2}}
$$

The drag force is found by integrating the pressure $\mathrm{p}$ and shear $\tau$ around the surface of the drop:

$$
F=-\int_{0}^{\pi} p\left(\theta, r_{d}\right) \cos \theta d A-\int_{0}^{\pi} \tau\left(\theta, r_{d}\right) \sin \theta d A
$$


where $d A=2 \pi r_{d}^{2} \sin \theta d \theta$. For $N_{R e} \ll 1$, Stokes (1851) showed that $C_{D}=24 / N_{R e}$ for a sphere falling in an unbounded fluid $\left(r_{\infty}=\infty\right)$, with one-third of the drag arising from pressure and twothirds from friction. For $N_{R e} \ll 1$ in a bounded fluid, Hamielec et al. (1967) showed that Stokes' representation of the stream function would yield $C_{D}^{a}=-32 B / N_{R e}$, where

$$
B=\frac{(15 / 2) r_{\infty}^{6}}{2-10 r_{\infty}^{3}+18 r_{\infty}^{5}-10 r_{\infty}^{6}}
$$

The significance of these analytical forms is that they provide a reference for comparison with the computed values for small Reynolds numbers.

Jenson (1959) showed that the components of the drag coefficient from friction $C_{D F}$ and pressure $C_{D P}$ may be determined as

$$
C_{D F}=\frac{8}{N_{R e}} \int_{0}^{\pi} \zeta \sin \theta d \theta
$$

and

$$
C_{D P}=\int_{0}^{\pi} p \sin 2 \theta d \theta
$$

where $p$ is the dimensionless (dynamic) pressure, $p=\left(p^{\prime}-p_{\infty}^{\prime}\right) /\left(\frac{1}{2} \rho U^{2}\right)$ and $p_{\infty}^{\prime}$ is the free stream static pressure. $C_{D F}$ may be readily computed from the vorticity distribution at the sphere surface determined while solving for the stream function. Following Jenson, the pressure distribution around the surface of the sphere is

$$
p(\theta)=p_{0}+\frac{4}{N_{R e}} \int_{0}^{\theta}\left(\zeta+\frac{\partial \zeta}{\partial r}\right) d \theta
$$

and the pressure at the forward stagnation point is

$$
p_{0}=1+\frac{8}{N_{R e}} \int_{1}^{\infty}\left(\frac{\partial \zeta}{\partial \theta}\right) \frac{d r}{r} .
$$

Calculated distributions of pressure and vorticity around the surface of the sphere are presented in Fig. 4 and Fig. 5, respectively, for $N_{R e}=5,10,20,40$, and 100. The pressure is maximum at the front stagnation point, then decreases around the sphere and becomes negative; that is, the total pressure is less than the static pressure far from the sphere. As the Reynolds number increases, the magnitude of the (dimensionless) pressure at the forward and rearward stagnation points decreases, as does the corresponding drag coefficient. 


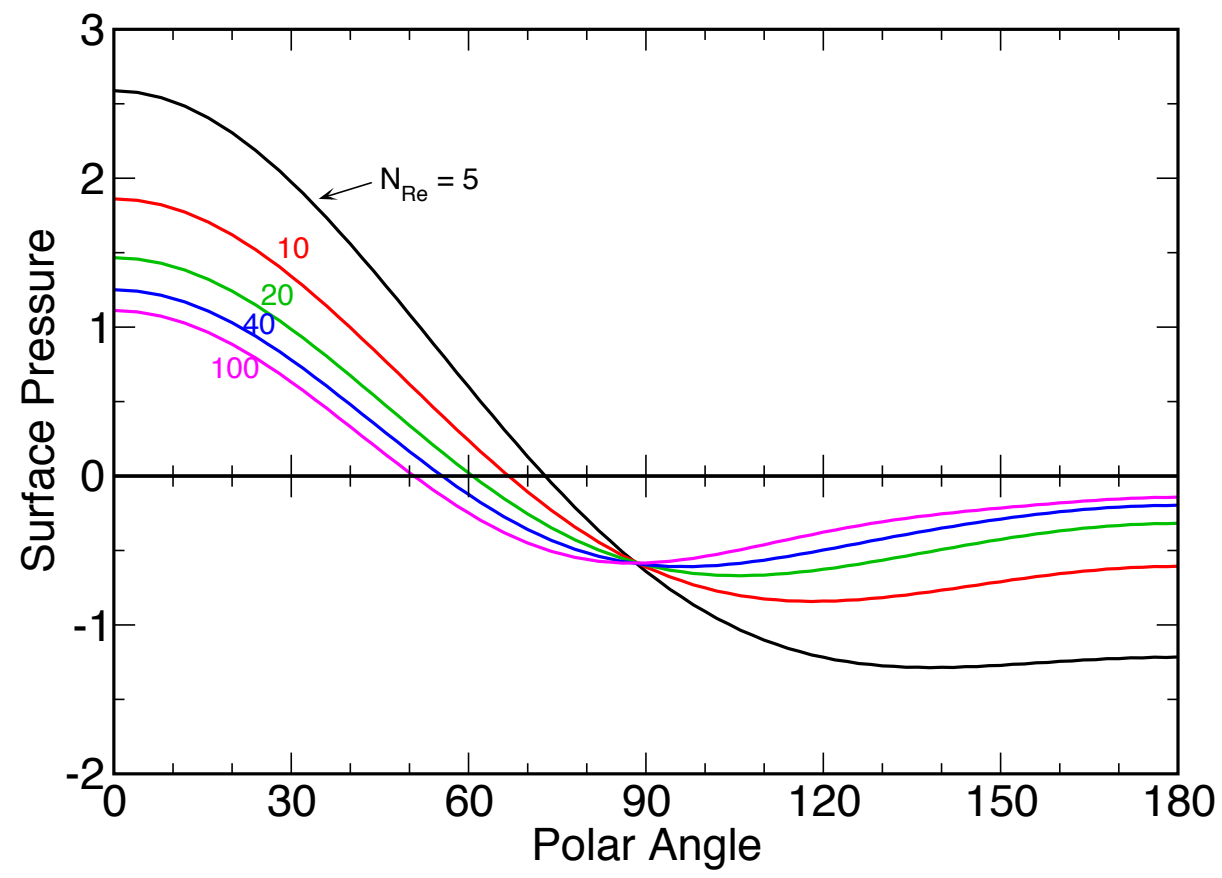

Figure 4 Calculated Surface Pressure Distributions around a Falling Drop

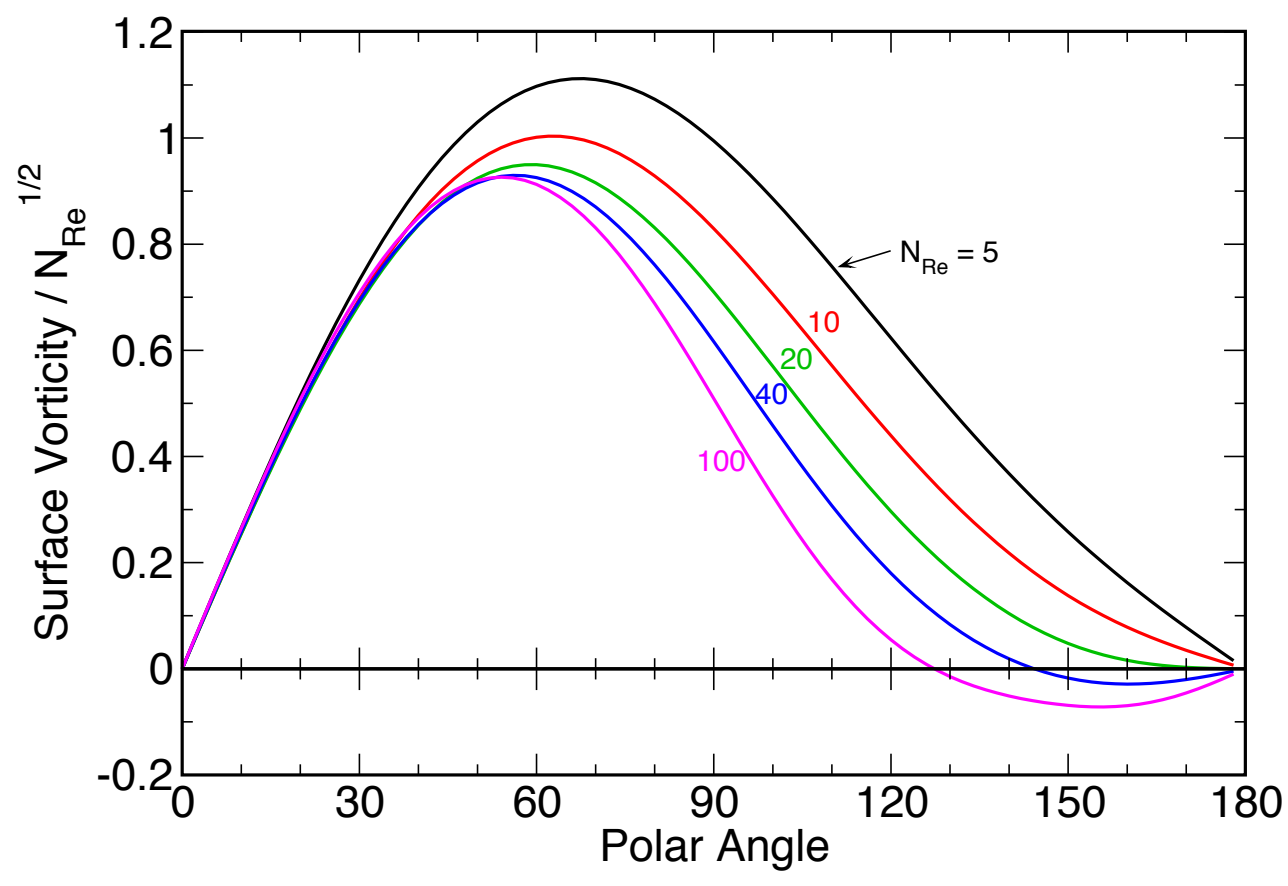

Figure 5 Calculated Surface Vorticity Distributions around a Falling Drop 
In contrast, the vorticity at the forward and rearward stagnation points is always zero, reaching a maximum where the streamlines are closest, as shown in Fig. 6. For $N_{R e}=40$ and 100, the vorticity becomes negative at the angle where the flow separates from the sphere and a recirculation region develops. This counter-rotating region is evident in the streamlines for these Reynolds numbers. The separation first occurs at $N_{R e}=22$, in agreement with LeClaire et al. (1970) and observations.

Table 2 shows that for $N_{R e}=0.01$ and $r_{\infty}=1808$, the calculated value of the drag coefficient $\left(C_{D}=2408\right)$ is very close to the analytical value for a bounded flow $\left(C_{D}{ }^{a}=2402\right)$ and to the corresponding Stokes (1851) value for an unbounded flow (2400). As the outer boundary is moved closer to the sphere, the drag coefficient increases as the influence of the boundary propagates to the sphere. However $C_{D}$ and $C_{D}{ }^{a}$ remain within $0.6 \%$. The sensitivity to the position of the outer boundary rapidly decreases as the Reynolds number increases because the effect of the boundary is increasingly swept downstream before reaching the surface of the sphere. For $N_{R e}=0.1$, the difference in calculated values of $C_{D}$ for $r_{\infty}=90$ and 20 is about $8 \%$; for $N_{R e}=1$, the difference is $2 \%$. It is important to point out that, as an integral measure, the drag coefficient magnifies small errors in the stream function and vorticity calculations. The derived velocities are therefore believed to be accurate for $r_{\infty}=20$ or 12 at the Reynolds number indicated in the table.

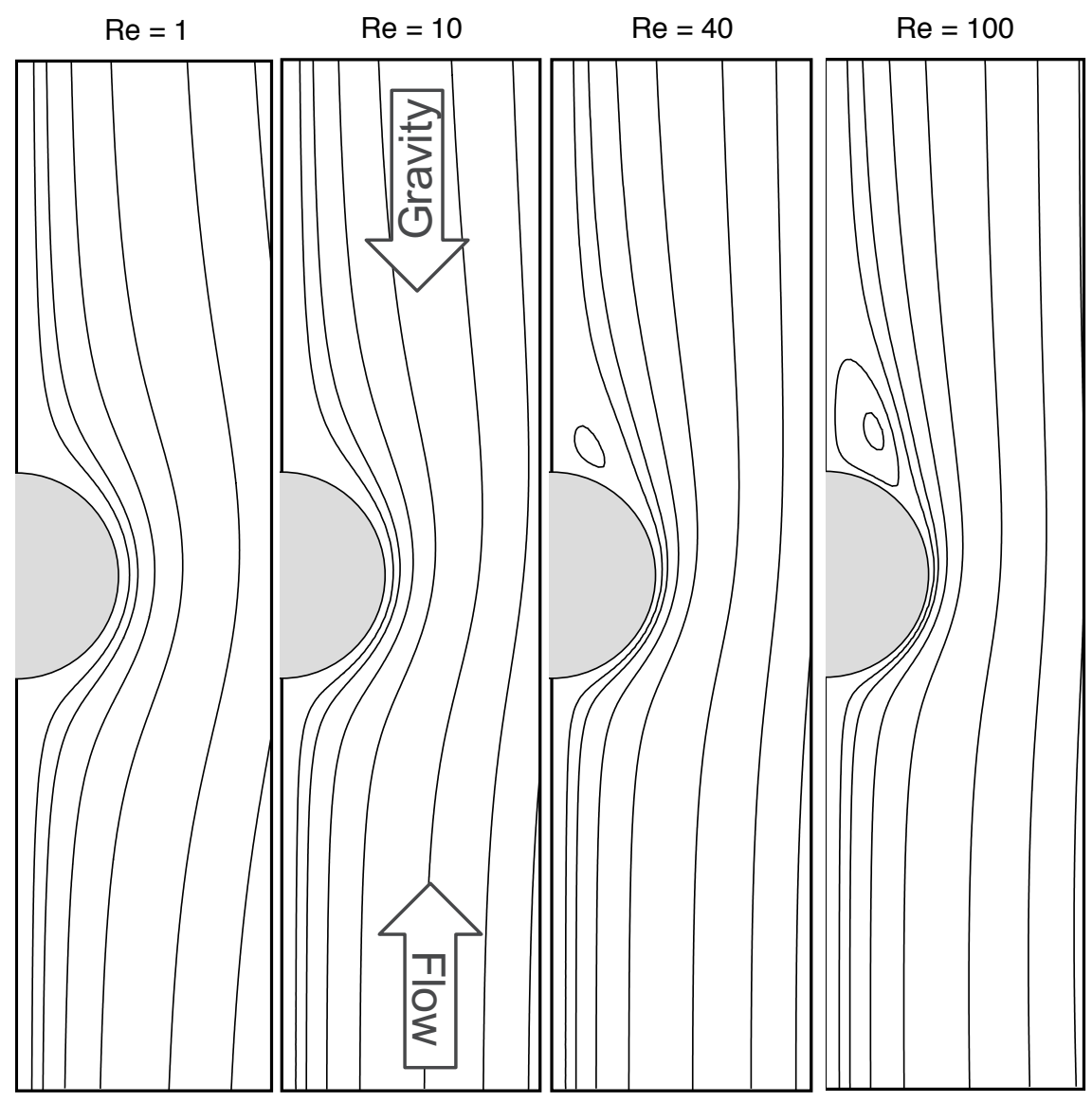

Figure 6 Streamlines of Calculated Stream Functions 
To validate these calculations, Fig. 7 presents a comparison of the present results with a function fitted to 480 experimentally determined values for $C_{D}$ over the range $2 \times 10^{-3} \leq N_{R e} \leq 2 \times 10^{5}$ (Cheng 2009):

$$
C_{D}=\frac{24}{N_{R e}}\left(1+0.26 N_{R e}\right)^{0.43}+0.47\left[1-\exp \left(-0.04 N_{R e}^{0.38}\right)\right] .
$$

Cheng reports the root-mean-square (RMS) error in the fit is 3.4\%. The fit is consistent with the Stokes (1851) limit for small Reynolds number, as shown. The present results demonstrate reasonable agreement with the fit, having an overall RMS error of 3.5\%. For $N_{R e} \leq 100$, the RMS error is $1.5 \%$. For $N_{R e} \geq 200$, the present results are increasingly low (almost $10 \%$ low at $N_{R e}=400$ ), likely because the actual wake flow becomes unsteady at these Reynolds numbers and is not fully represented in the present steady-flow approach.

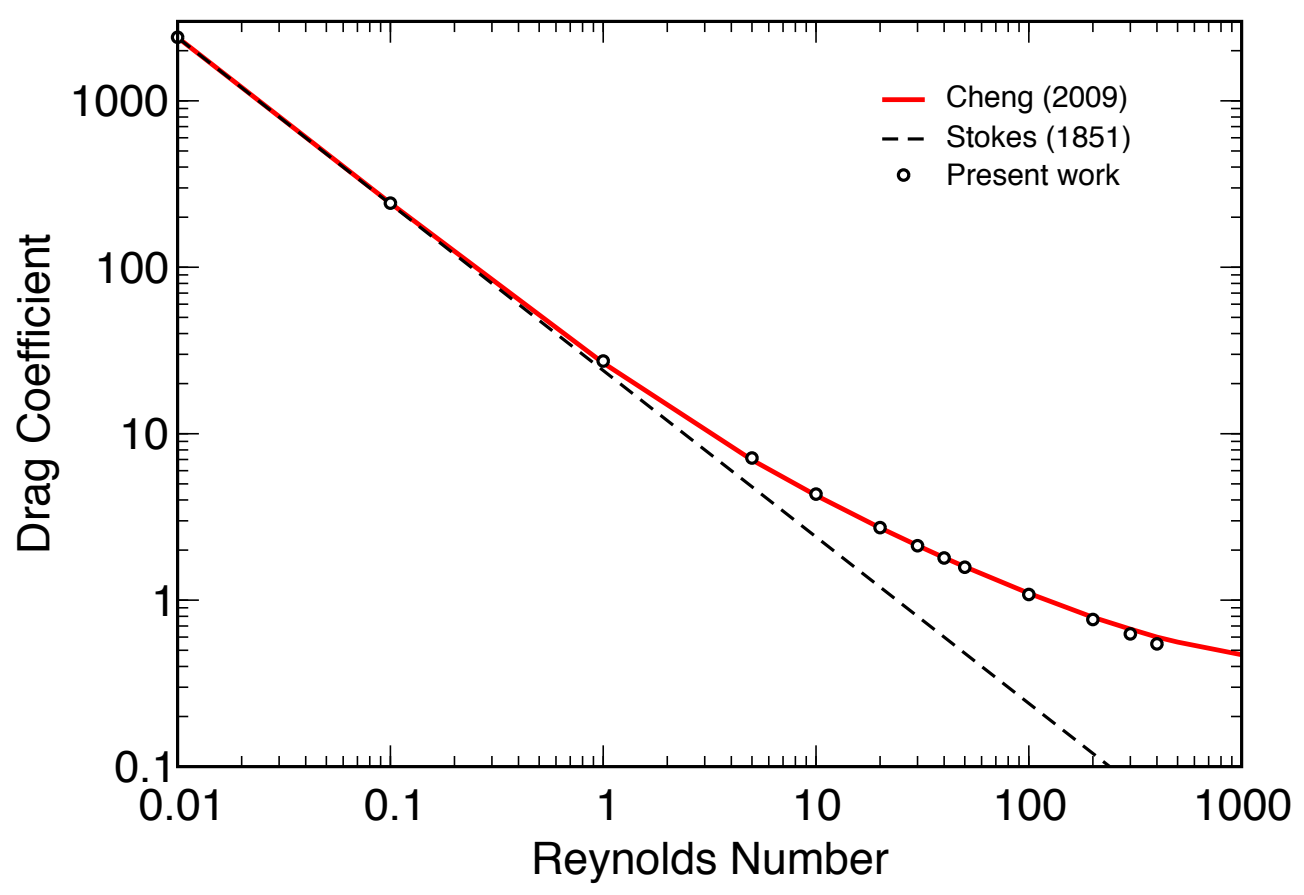

Figure 7 Drag Coefficients from the Present Work Compared with a Fit to Experimental Data (Cheng 2009) and the Stokes Limit 


\subsection{Recovering the Velocity Field}

At grid points interior to the boundaries, the radial and angular velocity components are calculated using central differences and $\partial \psi / \partial r=(\partial \psi / \partial z)(\partial z / \partial r)=1 / e^{z}(\partial \psi / \partial z)$ :

$$
\begin{aligned}
& U_{r}=\frac{-1}{r^{2} \sin \theta} \frac{\partial \psi}{\partial \theta}=\frac{-1}{e^{2 z_{j}} \sin \theta_{i}} \frac{\Psi_{i+1, j}-\psi_{i-1, j}}{2 \Delta \theta} \\
& U_{\theta}=\frac{1}{r \sin \theta} \frac{\partial \psi}{\partial r}=\frac{1}{e^{2 z_{j}} \sin \theta_{i}} \frac{\psi_{i, j+1}-\Psi_{i, j-1}}{2 \Delta z} .
\end{aligned}
$$

At the surface of the sphere, $U_{r}=U_{\theta}=0$.

The boundary condition at the outer boundary gives $U_{r}=-\cos \theta ; U_{\theta}=\sin \theta$; however, for consistency, the velocity components are computed from the stream function. The radial component is calculated as for an interior grid point; the angular component is

$$
U_{\theta}=\frac{1}{e^{2 z_{j}} \sin \theta_{i}} \frac{3 \psi_{i, N_{r}}-4 \psi_{i, N_{r}-1}+\psi_{i, N_{r}-2}}{2 \Delta z},
$$

where $N_{r}=1+\ln r_{\infty} / \Delta z$ is the number of radial grid points.

Along the plane of symmetry, $U_{\theta}=0$; however, because $\partial \psi / \partial \theta=0$ and $\sin \theta=0$, L'Hospital's (L'Hôpital's) Rule must be applied to find the radial component:

$$
\begin{aligned}
U_{r} & =\frac{-1}{r^{2} \cos \theta} \frac{\partial^{2} \psi}{\partial \theta^{2}} \\
& =\frac{-1}{e^{2 z_{j}}} \cdot \frac{2 \psi_{1, j}-5 \psi_{2, j}+4 \psi_{3, j}-\psi_{4, j}}{\Delta \theta^{2}} ; \theta=0^{\circ} \\
& =\frac{1}{e^{2 z_{j}}} \cdot \frac{2 \psi_{N_{\theta}, j}-5 \psi_{N_{\theta}-1, j}+4 \psi_{N_{\theta}-2, j}-\psi_{N_{\theta}-3, j}}{\Delta \theta^{2}} ; \theta=180^{\circ},
\end{aligned}
$$

where $N_{\theta}=1+\pi / \Delta \theta$ is the number of angular grid points.

In anticipation of the trajectory calculation, radial and angular velocity components are converted to rectilinear components according to

$$
\begin{aligned}
& U_{x}=U_{r} \cos \theta-U_{\theta} \sin \theta, \\
& U_{y}=U_{r} \sin \theta+U_{\theta} \cos \theta,
\end{aligned}
$$

which yields $U_{x}=-1, U_{y}=0$ at the outer boundary; $U_{x}=U_{y}=0$ at the surface of the sphere, and $U_{x}=U_{r}\left(\theta=0^{\circ}\right), U_{x}=-U_{r}\left(\theta=180^{\circ}\right), U_{y}=0$ along the plane of symmetry. 


\section{Trajectory Calculation}

\subsection{Equation of Motion of the Particle}

Finding the critical radius requires calculating the trajectory of the particle in the flow field around the drop by numerically integrating the dimensionless equation of motion of the particle subject to inertial, gravitational, and electrostatic forces (Beard and Grover 1974; Grover and Beard 1975):

$$
\frac{d \boldsymbol{V}}{d t}=\frac{\hat{\boldsymbol{z}}}{\mathrm{N}_{\mathrm{Fr}}}-\frac{1}{\mathrm{~K}}(\boldsymbol{V}-\boldsymbol{U})-\frac{F_{E}}{\mathrm{~N}_{\mathrm{Fr}}} \hat{\boldsymbol{r}}
$$

$\boldsymbol{V}$ is the velocity vector of the particle, $\boldsymbol{U}$ is the velocity vector of the flow around the drop, $\hat{\boldsymbol{z}}$ is a unit vector in the direction of gravity, $\hat{\boldsymbol{r}}$ is a unit vector directed from the center of the drop toward the center of the particle, $N_{F r}$ is the Froude number, $N_{F r}=U_{\infty}^{2} /\left(g^{*} r_{d}\right)$, which reflects the relative magnitude of inertial and gravitational forces, $g^{*}=g\left(1-\rho / \rho_{p}\right)$ is the net gravitational acceleration accounting for the buoyant force of air, $K$ is the Stokes number, $K=\left(r_{p} / r_{d}\right)^{2} \rho_{p} \mathrm{~N}_{\mathrm{Re}} \mathrm{C}_{\mathrm{sc}} /(9 \rho)$, which is the ratio of the response time of the particle to the characteristic time scale of the flow, indicating the propensity of the particle to follow a streamline, $C_{S C}$ is the Cunningham slip correction factor (Hinds 1982, p. 45), and $F_{E}$ is the dimensionless electrostatic force exerted on the particle by the drop: $F_{E}=F_{E}^{\prime} /\left(m_{p} g^{*}\right)$, where, as earlier, the prime indicates the dimensional quantity, and $m_{p}$ is the mass of the particle. Also as earlier, the velocities have been non-dimensionalized using the terminal fall speed of the drop and lengths have been non-dimensionalized using the radius of the drop.

Far from the drop, $d \boldsymbol{V} / d t \rightarrow 0$ and $F_{E} \rightarrow 0$ so that the equation of motion reduces to

$$
\boldsymbol{V}_{\infty}=\boldsymbol{U}_{\infty}+\frac{\mathrm{K}}{\mathrm{N}_{\mathrm{Fr}}} \hat{\mathbf{z}}
$$

Substituting this expression into the equation of motion yields

$$
\frac{d \boldsymbol{V}}{d t}=\frac{1}{\mathrm{~K}}\left[\left(\boldsymbol{V}_{\infty}-\boldsymbol{U}_{\infty}\right)-(\boldsymbol{V}-\boldsymbol{U})\right]-\frac{F_{E}}{\mathrm{~N}_{\mathrm{Fr}}} \hat{\boldsymbol{r}} .
$$

In rectilinear coordinates it is convenient to use the inertial reference frame of the falling drop with gravity aligned in the positive $x$ direction so that the vector equation of motion may be expressed as two scalar equations:

$$
\frac{d V_{x}}{d t}=\frac{1}{\mathrm{~K}}\left[\left(V_{\infty}+1\right)-\left(V_{x}-U_{x}\right)\right]-\frac{F_{E}}{\mathrm{~N}_{\mathrm{Fr}}} \cos \theta_{p}
$$




$$
\frac{d V_{y}}{d t}=\frac{-1}{\mathrm{~K}}\left(V_{y}-U_{y}\right)-\frac{F_{E}}{\mathrm{~N}_{\mathrm{Fr}}} \sin \theta_{p}
$$

where $V_{\infty}+1=K / N_{F r}, \theta_{p}=\tan ^{-1}\left(y_{p} / x_{p}\right)$, and $\left(x_{p}, y_{p}\right)$ is the coordinate location of the particle.

\subsection{Electrostatic Force on the Particle}

As noted by Grover and Beard (1975), in an exact treatment of the electrostatic force between a liquid drop and a particle, the drop would be represented by a sphere with a dielectric constant ranging from 81 (pure water) to infinity, depending on the composition of the liquid, and the particle would be represented by a smaller sphere with a dielectric constant ranging from 1 to infinity depending on the amount of water condensed on its surface. Lacking such a treatment, Grover and Beard pursed two conceptually opposite approaches. In the first approach, the drop and particle are represented as dielectric spheres with fixed point charges at their centers. In the second, they are represented by conducting spheres where the charges may freely redistribute on the surfaces of the spheres as they approach each other. The point charge approach is vastly simpler to implement, depending only on the product of the charges and the inverse square of the distance between them; however, it is only valid when both the drop and sphere are charged. The conducting spheres approach is complex, as shown below, but can accommodate the case where only one of the spheres is charged, and Davis (1969) has shown that representing a water drop as a conducting sphere is an excellent approximation of its electrical properties. Grover and Beard compared these approaches and found that, for cases involving charges on both spheres, they yielded very nearly identical results. For this reason the point charges approach has been utilized in the present work only to verify the implementation of the conducting spheres approach; it will not be further discussed. The computational requirements of the conducting spheres approach are easily accommodated by current desktop computers.

Davis (1964) solved for the force between two conducting spheres in an external electric field. In the absence of an external electric field, the force on one (here taken to be the particle) arising from charges on one or both spheres reduces to

$$
F_{E}=\frac{1}{\epsilon r_{p}^{2}}\left(F_{5} Q_{d}^{2}+F_{6} Q_{d} Q_{p}+F_{7} Q_{p}^{2}\right)
$$

In this expression all quantities are dimensional (cgs system) per Davis. $Q$ is the charge on the particle or drop in electrostatic units (esu), $\epsilon$ is the dielectric constant of air $\left(=1.00059\right.$ at $\left.25^{\circ} \mathrm{C}\right)$, and the force coefficients $F_{5}, F_{6}$, and $F_{7}$ are defined by Davis as

$$
F_{j}=\kappa_{j} \sum_{k=1}^{24} \mathrm{~K}_{j k} f_{k}+\beta_{j} ; j=1, \ldots, 10
$$


where $\kappa_{j}$ are simple factors (Davis, Table 2 ), $\mathrm{K}_{j k}$ are relatively simple polynomial expressions (Davis, Table 4), $\beta_{j}$ are either 0 or -1 (Davis, Table 2 ), and $f_{k}$ are series of type $T_{m}$ or $U_{m}$ (Davis, Table 3):

$$
\begin{gathered}
T_{m}(\xi)=\sum_{n=0}^{k-1} \frac{(2 n+1)^{m} e^{(2 n+1) \xi}}{\left(e^{(2 n+1) b}-1\right)^{2}}+\sum_{v=0}^{\infty}(v+1) \lambda_{v}(m) ; \\
U_{m}(\xi)=\sum_{n=0}^{k-1} \frac{(2 n+1)^{m} e^{(2 n+1) \xi}}{\left(e^{(2 n+1) b}-1\right)\left(e^{(2 n+3) b}-1\right)}+\sum_{v=0}^{\infty} \varsigma_{v} \lambda_{v}(m),
\end{gathered}
$$

where

$$
\zeta_{v}=\sum_{r=1}^{v+1} e^{-2 r b}
$$

$\lambda_{\nu}(m)$ are polynomial expressions (Davis, eq. 49) and $b=\mu_{1}+\mu_{2}$, where $\mu$ characterizes the sphere in the bi-spherical coordinate system Davis employed:

$$
\begin{gathered}
\mu_{1}=\ln \left(\frac{D_{1}+a}{R_{1}}\right) ; \mu_{2}=\ln \left(\frac{D_{2}+a}{R_{2}}\right) ; \\
a=\left(D_{1}^{2}-R_{1}^{2}\right)^{1 / 2}=\left(D_{2}^{2}-R_{2}^{2}\right)^{1 / 2} ; \\
D_{1}=\left(h^{2}+R_{1}^{2}-R_{2}^{2}\right) /(2 h) ; D_{2}=h-D_{1},
\end{gathered}
$$

where $D$ is the distance from the coordinate origin to the center of the sphere, $R$ is the sphere radius, and $h$ is the distance between the centers of the spheres. In his Appendix A, Davis provides guidance on the summation of these series. The correct implementation of Davis's solution was verified by comparing the calculated force coefficients with the tabulated values (Davis, Table 1) for a range of $R_{1} / R_{2}$ and $s / R_{2}$ where $s=h-\left(R_{1}+R_{2}\right)$ is the separation distance between the surface of the spheres.

Grover and Beard (1975) cite several experimental studies to show that a typical mean observed drop charge for thunderstorms or warm rain is $\left|Q_{d}\right| / r_{d}^{2} \lesssim 2$ for charge in esu and radius in cm, and the maximum observed drop charge in thunderstorms is $\left|Q_{d}\right| / r_{d}^{2} \lesssim 20$, which they note is approximately $1 / 5$ of the corona discharge limit.

\subsection{Phoretic Forces on the Particle}

In subsequent work, Grover et al. (1977) and Wang et al. (1978) included phoretic forces (thermophoresis and diffusiophoresis) in their trajectory calculations. Because phoretic effects are important primarily for sub-micron particles, those effects have not been included here, since 
the particle sizes of candidate biological agents for knockdown spray mitigation are greater than $1 \mu \mathrm{m}$. However, phoretic effects could be added without significant incremental effort, if needed.

\subsection{Finding the Fluid Velocity at the Particle Position}

At each step in the trajectory calculation, the velocity components of the fluid must be determined at the particle location. To accomplish this, an interpolation scheme (Anderson et al. 1984, p. 57) has been implemented as follows. Assume that the local variation of the velocity components at any point may be described by a polynomial form:

$$
f(\theta, z)=f_{0}(\theta, z)+f_{1}(\theta, z)+f_{2}(\theta, z)+\cdots+f_{n}(\theta, z)
$$

where

$$
f_{n}(\theta, z)=c_{n, 0} \theta^{n}+c_{n, 1} \theta^{n-1} z+\cdots+c_{n, n-1} \theta z^{n-1}+c_{n, n} \theta^{n} z^{n}
$$

For $n=2$, this becomes

$$
f(\theta, z)=c_{0,0}+c_{1,0} \theta+c_{1,1} z+c_{2,0} \theta^{2}+2 c_{2,1} \theta z+c_{2,2} z^{2} .
$$

To find the coefficients, carry out a Taylor series expansion centered on a nearby grid location:

$\left.\left.\left.\left.\left.f(\delta \theta, \delta z)=f(0,0)+\frac{\partial f}{\partial \theta}\right)_{0,0} \delta \theta+\frac{\partial f}{\partial z}\right)_{0,0} \delta z+\frac{1}{2} \frac{\partial^{2} f}{\partial \theta^{2}}\right)_{0,0} \delta \theta^{2}+\frac{\partial^{2} f}{\partial \theta \partial z}\right)_{0,0} \delta \theta \delta z+\frac{1}{2} \frac{\partial^{2} f}{\partial z^{2}}\right)_{0,0} \delta z^{2}$.

Then

$\left.\left.\left.\left.\left.f(0,0)=c_{0,0} ; \frac{\partial f}{\partial \theta}\right)_{0,0}=c_{1,0} ; \frac{\partial f}{\partial z}\right)_{0,0}=c_{1,0} ; \frac{\partial^{2} f}{\partial \theta \partial z}\right)_{0,0}=2 c_{2,1} ; \frac{1}{2} \frac{\partial^{2} f}{\partial \theta^{2}}\right)_{0,0}=c_{2,0} ; \frac{1}{2} \frac{\partial^{2} f}{\partial z^{2}}\right)_{0,0}=c_{2,2}$.

In terms of the gridded values centered on $f_{i, j}\left(=c_{0,0}\right)$

$$
\begin{aligned}
& f_{i+1, j}=c_{0,0}+c_{1,0} \Delta \theta+c_{2,0} \Delta \theta^{2} ; f_{i-1, j}=c_{0,0}-c_{1,0} \Delta \theta+c_{2,0} \Delta \theta^{2} \\
& f_{i, j+1}=c_{0,0}+c_{1,1} \Delta z+c_{2,2} \Delta z^{2} ; f_{i, j-1}=c_{0,0}-c_{1,1} \Delta z+c_{2,2} \Delta z^{2} ; \\
& f_{i+1, j+1}=c_{0,0}+c_{1,0} \Delta \theta+c_{1,1} \Delta z+c_{2,0} \Delta \theta^{2}+2 c_{2,1} \Delta \theta \Delta z+c_{2,2} \Delta z^{2}
\end{aligned}
$$

which can be solved for the coefficients:

$$
\begin{aligned}
& c_{1,0}=\left(f_{i+1, j}-f_{i-1, j}\right) /(2 \Delta \theta) ; \\
& c_{1,1}=\left(f_{i, j+1}-f_{i, j-1}\right) /(2 \Delta z) ;
\end{aligned}
$$




$$
\begin{aligned}
& c_{2,0}=\left(f_{i+1, j}-2 f_{i, j}+f_{i-1, j}\right) /\left(2 \Delta \theta^{2}\right) ; \\
& c_{2,2}=\left(f_{i, j+1}-2 f_{i, j}+f_{i, j-1}\right) /\left(2 \Delta z^{2}\right) ; \\
& c_{2,1}=\left(f_{i+1, j+1}+f_{i, j}-f_{i+1, j}-f_{i-1, j}\right) /(2 \Delta \theta \Delta z) .
\end{aligned}
$$

Then the interpolated value is:

$$
f(\delta \theta, \delta z)=c_{0,0}+c_{1,0} \delta \theta+c_{1,1} \delta z+c_{2,0} \delta \theta^{2}+2 c_{2,1} \delta \theta \delta z+c_{2,2} \delta z^{2},
$$

where $\delta z=z_{p}-z_{j} ; \delta \theta=\theta_{p}-\theta_{i}$. The appropriate grid location $(i, j)$ is determined by $i=\operatorname{Integer}\left(z_{p} / \Delta z\right)+1 ; j=\operatorname{Integer}\left(\theta_{p} / \Delta \theta\right)+1$, where $z_{p}=\log \left(r_{p}\right)$. However, if $\delta z \rightarrow \Delta z$ and/or $\delta \theta \rightarrow \Delta \theta$, then the accuracy of the interpolation scheme is reduced in that dimension. To prevent this, if $\delta \theta / \Delta \theta>0.5, i=i+1$ or if $\delta z / \Delta z>0.5, j=j+1$, which keeps the interpolation centered on the particle location: $-0.5 \leq \delta \theta / \Delta \theta \leq 0.5$, thereby maintaining the accuracy of the scheme.

\subsection{Finding the Critical Radius}

The particle is initially placed at $r_{\infty}$, offset one drop radius from the plane of symmetry: $y_{p 0}=1, x_{p 0}=\left(r_{\infty}^{2}-1\right)^{1 / 2}$. Then the expressions for $d V_{x} / d t, d V_{y} / d t, d x_{p} / d t=V_{x}$, and $d y_{p} / d t=V_{y}$ are simultaneously integrated using a $5^{\text {th }}$-order Runge-Kutta algorithm with adaptive step size control (Press et al. 1992, p. 708), advancing the particle trajectory until either it collides with the drop (i.e., the separation distance between it and the drop are equal to the sum of their radii) or misses the drop. Then $y_{\mathrm{p} 0}$ is adjusted (increased for a collision, decreased for a miss), and the process is iterated until the difference between $y_{\mathrm{p} 0}$ for a miss and a subsequent collision are within $0.1 \mu \mathrm{m}$, then $r_{\mathrm{c}}=y_{\mathrm{p} 0}$ and the collision efficiency is calculated as described earlier.

\subsection{Results of the Trajectory Calculations}

Grover and Beard (1975) present calculated efficiencies for combinations of charges on the particle and drop over a range of particle and drop sizes. The efficiencies derived from the present work have been compared with theirs to verify the accuracy of the trajectory calculations. An example of the trajectory calculations and the collision efficiencies derived from them is presented in Fig. 8 for several combinations of electrostatic charge $(q=Q / r)$. With no charge on either the drop or particle, almost all particles follow the flow around the drop and the collision efficiency is very small $(E=0.001)$. As charge is added to the drop, particles are captured in the low-pressure region in the rear of the drop and the collision efficiency increases. When both particles are charged, the electrostatic force increases substantially because all three terms in the expression for the electrostatic force are contributing and particles starting several drop radii away are captured. 


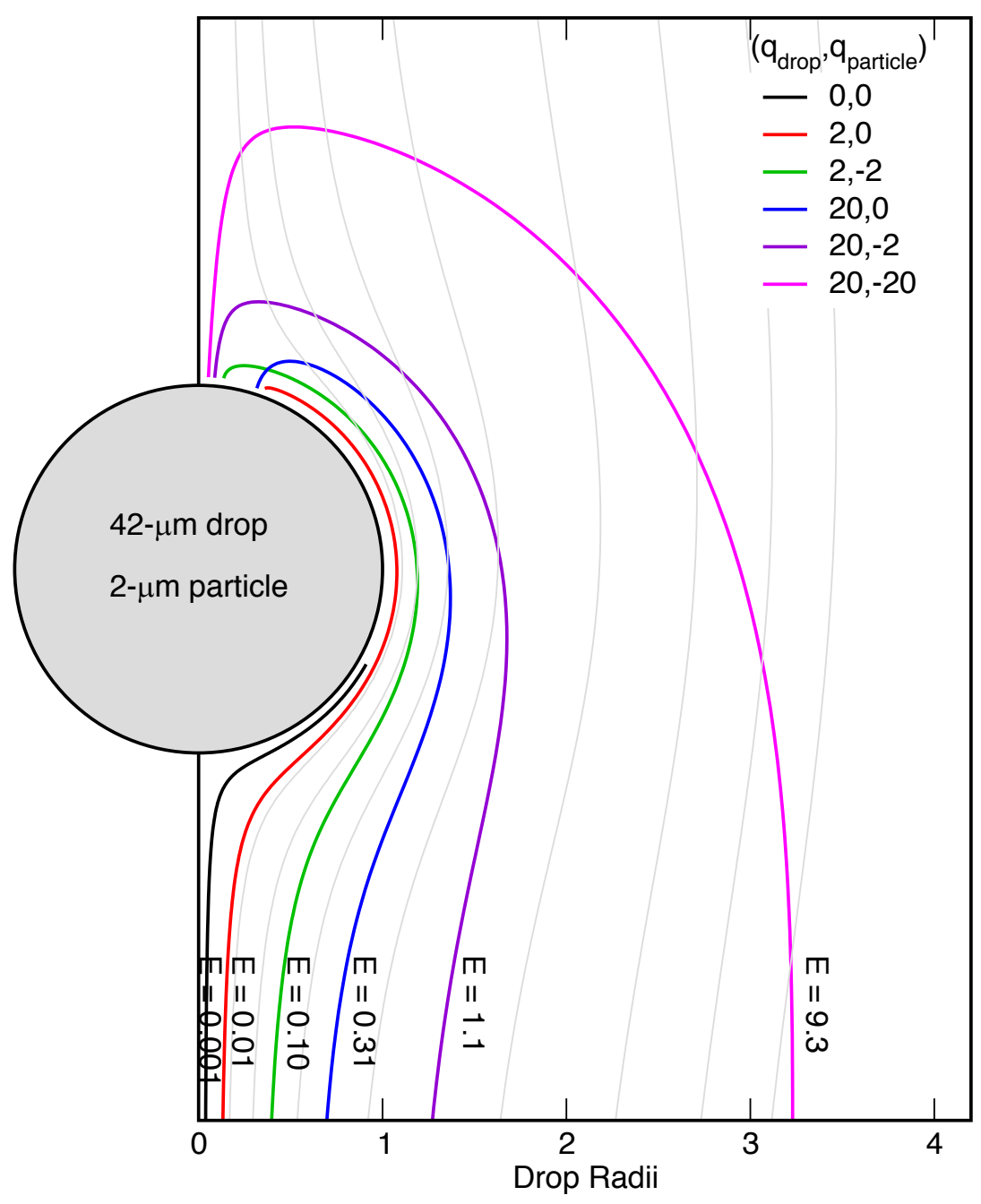

Figure 8 Trajectories and Collision Efficiencies for a 42- $\mu \mathrm{m}$-Diameter Drop and $2-\mu \mathrm{m}$-Diameter Particle for Several Combinations of Electrostatic Charge (Streamlines associated with the flow are also shown [thin gray lines]) 


\section{Comparison with Experiment}

Members of the technical staff at Sandia National Laboratories have carried out experiments with knockdown sprays (Tucker et al. 2016) in a test chamber measuring $8 \mathrm{ft}(244 \mathrm{~cm})$ on a side $\left(512 \mathrm{ft}^{3}, 14.5 \mathrm{~m}^{3}\right.$ volume). Spores of Bacillus atrophaeus $(\mathrm{Bg})$ were aerosolized ${ }^{2}$ in the chamber at a concentration of $10^{9} \mathrm{cfu} / \mathrm{m}^{3}$. After mixing for 120 minutes to achieve a uniform spatial distribution of $\mathrm{Bg}$, a decontaminant fluid (DF-200) ${ }^{3}$ developed by Sandia was sprayed into the chamber for 1 minute through nine electrostatic spray nozzles. Prior characterization of the nozzles (Betty et al. 2010) suggested that the drop diameter was in the range 30-40 $\mu \mathrm{m}$ at the nozzle pressure used in the test. The terminal fall speed of drops in this size range would be about $3-5 \mathrm{~cm} / \mathrm{s}$, requiring $50-80 \mathrm{~s}$ to fall to the chamber floor from the nozzle height, so nearly all of the drops would be airborne during the spray period and the contact time may be taken to be $60 \mathrm{~s}$. Tucker et al. (2016) reported a drop mass concentration of $138 \mathrm{~g} / \mathrm{m}^{3}$, which agrees with the total volume of DF-200 sprayed (2 L) divided by the chamber volume (assuming a density of $1 \mathrm{~g} / \mathrm{m}^{3}$ for DF-200). Tucker et al. (2016) also reported results for drop mass concentrations of 92 $\mathrm{g} / \mathrm{m}^{3}$ and $46 \mathrm{~g} / \mathrm{m}^{3}$, corresponding to $1.3 \mathrm{~L}$ and $0.67 \mathrm{~L}$ of DF-200 sprayed. For drop concentrations of $138 \mathrm{~g} / \mathrm{m}^{3}$ and $92 \mathrm{~g} / \mathrm{m}^{3}$, Tucker et al. (2016) reported a $5 \mathrm{log}$ reduction (e.g., 10 ${ }^{-5}$ ) in airborne Bg spores immediately after the spray ended and a $4 \log$ reduction for $46 \mathrm{~g} / \mathrm{m}^{3}$. These correspond to filter efficiencies of 0.99999 and 0.9999 , respectively.

Because neither the charge on the DF-200 drops nor the Bg spores were reported, calculated filter efficiencies are presented in Table 3 for several combinations of charge using the singledrop efficiencies in Fig. 8 for a $42-\mu \mathrm{m}$ diameter drop and 2- $\mu \mathrm{m}$ diameter particle. Comparing the calculated efficiencies with Sandia's observations suggests that there probably was a substantial charge on the DF-200 drops, though perhaps not as high as $20 \mathrm{esu} / \mathrm{cm}^{2}$, and that there was probably an opposite charge on the Bg spores, likely imparted by the aerosolization process.

Table 3 Calculated Filter Efficiencies for Comparison with a Knockdown Spray Experiment

\begin{tabular}{cccccc}
\hline & & & \multicolumn{3}{c}{ Filter Efficiency } \\
\cline { 4 - 6 }$q_{d}$ & $q_{p}$ & $E$ & $C_{d}=138 \mathrm{~g} / \mathrm{m}^{3}$ & $C_{d}=92 \mathrm{~g} / \mathrm{m}^{3}$ & $C_{d}=46 \mathrm{~g} / \mathrm{m}^{3}$ \\
\hline & & & & & \\
0 & 0 & 0.001 & 0.014 & 0.0096 & 0.0048 \\
2 & 0 & 0.01 & 0.13 & 0.092 & 0.047 \\
2 & -2 & 0.10 & 0.76 & 0.62 & 0.38 \\
20 & 0 & 0.31 & 0.99 & 0.95 & 0.78 \\
20 & -2 & 1.1 & 0.999999 & 0.99998 & 0.998 \\
20 & -20 & 9.3 & 1.0 & 1.0 & 1.0 \\
\hline
\end{tabular}

2 Although the particle size of the Bg aerosol was not reported, it was reported that the Bg was "weaponized-like." Accordingly a mass median aerodynamic dimeter of $2 \mu \mathrm{m}$ is assumed for the purpose of this comparison.

3 DF-200 is a mixture of a hydrogen peroxide solution, foaming components, and a "novel activator." 
Two additional points concerning the applicability of the drop spray model to the circumstances of the chamber tests are worth noting. First, the idealization of the single-drop efficiency implicitly assumes that the flow field around the drop is not affected by other drops; that is, the spacing between drops is assumed to be greater than twice the distance to the outer boundary used in the stream function calculations. The mean spacing between drops may be estimated from the mean volume occupied by a single drop:

$$
\bar{d}=(6 \bar{v} / \pi)^{1 / 3}
$$

where the mean volume occupied by a single drop is the inverse of the drop number concentration:

$$
\overline{\mathcal{V}}=N_{d}^{-1}=m_{d} / C_{d}
$$

and, as before, $C_{d}$ is the drop mass concentration and $m_{d}$ is the mass of a single drop. For the chamber studies, $\bar{d} / r_{d}=39$ for $C_{d}=138 \mathrm{~g} / \mathrm{m}^{3}$, which is about twice the distance to the outer boundary used to calculate the flow field around the drop; therefore, the assumption of isolated drops appears to be justified.

Another implicit assumption is that the impact of the particle does not affect the motion of the drop. Beard and Grover (1974) interpret this as requiring that the ratio of the mass of the particle to the mass of the drop be no larger than $10^{-3}$, which appears to be satisfied in the chamber tests, albeit without direct measurements of the $\mathrm{Bg}$ particle size. For cases where the particle and drop have different densities, the ratio of their diameters should be no larger than $0.1\left(\rho_{d} / \rho_{p}\right)^{1 / 3}$. 


\section{Concluding Remarks}

Models originally developed many years ago for industrial or atmospheric science purposes have been disinterred and implemented to calculate the flow field around a falling drop and the trajectory of a particle in that flow field to determine their collision efficiency as a function of their size, concentration, and electric charge. In the present case, the collision efficiency is a key parameter in our model for predicting the performance of a drop spray to mitigate the release of biological agent particles in a subway or other confined space.

The flow-field and trajectory models have been verified against the results presented in the original papers. The flow-field model has been validated against recent experimentally derived drag coefficients, albeit vicariously via a function fitted to the data. The drop-spray model, utilizing calculated collision efficiencies, has been compared with data obtained from tests of a knockdown spray. Despite the uncertainties in some of the test parameters, the comparison suggests that the drop-spray model may be able to accurately estimate the performance of a charged knockdown spray, and that the model may be useful in refining the design of such a spray for mitigation of a biological agent release in an indoor environment. 
This page intentionally left blank. 


\section{References}

Anderson D.A., J.C. Tannehill and R.H. Pletcher, 1984, Computational Fluid Mechanics and Heat Transfer, Hemisphere, New York, NY.

Beard, K.V., and S.N. Grover, 1974, "Numerical collision efficiencies for small raindrops colliding with micron size particles," J. Atmos. Sci., 31:543-550.

Betty R.G., D.A. Lucero, J. Brockmann, and M.D. Tucker, et al., 2010, Release Mitigation Spray Safety System for Chemical Demilitarization Applications, Report No. SAND2010-4023, June, Sandia National Laboratories Albuquerque, NM.

Cheng, L., 1973, "Collection of airborne dust by water sprays," Ind. Eng. Chem. Process Des. Develop., 12(3):221-225.

Cheng, N.S., 2009, "Comparison of formulas for drag coefficient and settling velocity of spherical particles.” Powder Technology, 189:395-398.

Davis, M.H., 1964, Two Charged Spherical Conductors in a Uniform Electric Field: Forces and Field Strength, Report RM-3860-PR, RAND Corporation, Santa Monica, CA.

Davis, M.H., 1969, "Electrostatic field and force on a dielectric sphere near a conducting plane - a note on the application of electrostatic theory to water droplets," Amer. J. Phys., 37:2629.

Grover, S.N., and K.V. Beard, 1975, “A numerical determination of the efficiency with which electrically charged cloud drops and small raindrops collide with electrically charged spherical particles of various densities," J. Atmos. Sci. 32:2156-2165.

Grover, S.N., H.R. Pruppacher, and A.E. Hammielec, 1975, "A numerical determination of the efficiency with which spherical aerosol particles collide with spherical water drops due to inertial impaction and phoretic and electrical forces," J. Atmos. Sci., 34:1655-1663.

Hamielec, A.E., T.W. Hoffmann, and L.L. Ross, 1967, "Numerical solution of the Navier-Stokes equation for flow past spheres: Part I. viscous flow around spheres with and without radial mass efflux," A.I.Ch.E. Journal, 13(2):212-219.

Hamielec, A.E., S.H. Storey, and J.M. Whitehead, 1963, "Viscous flow around fluid spheres at intermediate Reynolds number (II)," Can. J. Chem. Eng., 41(6):246-251.

Hinds, W.C., 1982, Aerosol Technology, Wiley, New York, NY, Wiley.

Jenson, V.G., 1959, "Viscous flow round a sphere at low Reynolds numbers $(<40)$," Proc. Roy. Soc. London, 249A:346-366. 
LeClaire, B.P., A.E. Hamielec, and H.R. Pruppacher, 1970, "A numerical study of the drag on a sphere at low and intermediate Reynolds numbers," J. Atmos. Sci., 27:308-315.

Press, W.H., S.A. Teukolsky, W.T. Vetterling, and B.P. Flannery, 1992, Numerical Recipes in FORTRAN: The Art of Scientific Computing, $2^{\text {nd }}$ ed., Cambridge University Press, Cambridge, MA.

Prostanski, D., 2013, "Use of air-and-water spraying systems for improving dust control in mines,” J. Sust. Min. 12(2):29-34.

Pruppacher, H.R., R.G. Semonin, and W.G.N. Slinn, eds. 1983, Precipitation Scavenging, Dry Deposition, and Resuspension, Volume 1. Elsevier; New York, NY.

Pruppacher, H.R., and J.D. Klett, 2010, "Cloud particle interactions," in: Microphysics of Clouds and Precipitation, $2^{\text {nd }}$ Edition, Springer, New York, NY, 568-616, 846-852.

Schowengerdt, F.D., and J.T. Brown, 1976, "Colorado School of Mines tackles control of respirable coal dust," Coal Age, April.

Slinn, W.G.N., 1984, "Precipitation scavenging," in: Randerson D., ed. Atmospheric Science and Power Production, U.S. Department of Energy. Technical Information Service, Springfield, VA, 466-532.

Stokes, G.G., 1851, "On the effect of the internal friction of fluids on the motion of pendulums," Trans. Camb. Phil. Soc., 9(2):8-106.

Tucker, M.D., A.L. Sanchez, C.A. Brusseau, and M.S. Tezak, et al., 2016, Spray Knockdown System for Rapid Containment and Neutralization of Airborne CBW Agents, Presentation No. SAND2016-11052C, Sandia National Laboratories, Albuquerque, NM.

Wang, P.K., S.N. Grover, and H.R. Pruppacher, 1978, "On the effect of electric charges on the scavenging of aerosol particles by clouds and small raindrops," J. Atmos. Sci., 35:1735-1743.

White, F.M., 1974, Viscous Fluid Flow, McGraw-Hill, New York, NY. 



\section{Argonne}

Strategic Security Sciences Division

9700 South Cass Avenue, Bldg. 221

Argonne, IL 60439-4854

www.anl.gov

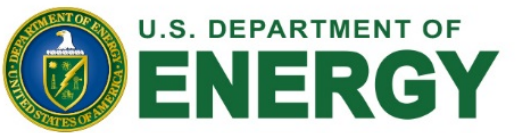

Argonne National Laboratory is a U.S. Department of Energy

laboratory managed by UChicago Argonne, LLC 